\title{
CORRECTION
}

\section{Correction to: Ambulatory blood pressure profiles in familial dysautonomia}

\author{
Lior Goldberg ${ }^{1,3}$ (I) $\cdot$ Bat-El Bar-Aluma ${ }^{1,3} \cdot$ Alex Krauthammer $^{1,3} \cdot$ Ori Efrati $^{1,3} \cdot$ Yehonatan Sharabi $^{2,3}$
}

Published online: 4 April 2020

○) Springer-Verlag GmbH Germany, part of Springer Nature 2020

Correction to: Clinical Autonomic Research (2018) 28:385-390 https://doi.org/10.1007/s10286-018-0507-1

Unfortunately, the original version of this article contained a mistake in the first Author's ORCID ID.

The corrected ORCID ID is: http://orcid.org/0000-00021643-7616.

The original article can be found online at https://doi.org/10.1007/ s10286-018-0507-1.

Bat-El Bar-Aluma

BatEl.BarAluma@sheba.health.gov.il

1 Pediatric Pulmonary Unit and The National Center for Familial Dysautonomia, Edmond and Lily Safra Children's Pediatric Hospital, Chaim Sheba Medical Center, Tel-HaShomer, Israel

2 Hypertension Unit, Chaim Sheba Medical Center, Tel-HaShomer, Israel

3 Sackler Faculty of Medicine, Tel Aviv University, Tel Aviv, Israel 